\title{
EFEITO DA VELOPLASTIA INTRAVELAR SOBRE A NASALIDADE EM INDIVÍDUOS COM INSUFICIÊNCIA VELOFARÍNGEA
}

\section{Effect of intravelar veloplasty on nasality in patients with velopharyngeal insufficiency}

\author{
Renata Paciello Yamashita ${ }^{(1)}$, Ester Luisa Leite Carvalho (2), Ana Paula Fukushiro ${ }^{(3)}$, \\ Neivo Luiz Zorzetto ${ }^{(4)}$, Inge Elly Kiemle Trindade ${ }^{(5)}$
}

\begin{abstract}
RESUMO
Objetivo: verificar o efeito da palatoplastia secundária realizada com veloplastia intravelar sobre a nasalidade e nasalância dos pacientes com fissura de palato reparada e insuficiência velofaríngea (IVF) e comparar os resultados entre estes pacientes, de acordo com o grau de fechamento velofaríngeo aferido na nasofaringoscopia. Método: estudo prospectivo com 40 pacientes de ambos os sexos, com idades entre 4 e 48 anos, com fissura de palato reparada e IVF residual, avaliado 3 dias antes e 8 meses após a palatoplastia, em média, divididos em dois grupos: um com 25 pacientes com falhas pequenas (grupo I) e outro com 15 pacientes com falhas médias a grandes (grupo II) no fechamento velofaríngeo. A hipernasalidade foi avaliada perceptivamente e nasalância foi avaliada por meio da nasometria. Diferenças entre grupos e etapas foram consideradas significativas ao nível de $5 \%$. O estudo foi aprovado pelo Comitê de Ética para Pesquisa com Seres Humanos da Instituição. Resultados: após a cirurgia, verificou-se redução da hipernasalidade em $84 \%$ dos pacientes do grupo I e em $73 \%$ dos pacientes do grupo II. Redução da nasalância foi observada em $52 \%$ dos casos do grupo I e em $43 \%$ dos pacientes do grupo II. Conclusão: a palatoplastia secundária com veloplastia intravelar levou à melhora da nasalidade na maioria dos pacientes analisados. Os resultados também demonstraram que a cirurgia foi mais efetiva nos pacientes que apresentavam falhas pequenas no fechamento velofaríngeo.
\end{abstract}

DESCRITORES: Fala; Fissura Palatina; Insuficiência Velofaríngea

(1) Fonoaudióloga; Orientadora do programa de Pós Graduação em Ciências da Reabilitação do Hospital de Reabilitação de Anomalias Craniofaciais da Universidade de São Paulo (HRAC-USP); Chefe do Laboratório de Fisiologia do HRAC-USP; Doutora em Ciências pelo programa de Pós Graduação em Ciências do HRAC-USP, Bauru, São Paulo, Brasil.

(2) Fonoaudióloga; Doutora em Ciências da Reabilitação pelo programa de Pós-Graduação em Ciências da Reabilitação do Hospital de Reabilitação de Anomalias Craniofaciais da Universidade de São Paulo (HRAC-USP), Bauru, São Paulo, Brasil.

(3) Fonoaudióloga; Fonoaudióloga do Laboratório de Fisiologia do HRAC-USP, Bauru, São Paulo, Brasil; Professora doutora do Departamento de Fonoaudiologia da Faculdade de Odontologia de Bauru da Universidade de São Paulo (FOB-USP), Bauru, São Paulo, Brasil; Orientadora do programa de Pós Graduação em Ciências da Reabilitação do Hospital de Reabilitação de Anomalias Craniofaciais da Universidade de São Paulo (HRAC-USP); Doutora em
Ciências da Reabilitação pelo programa de Pós-Graduação em Ciências da Reabilitação do HRAC-USP, Bauru, São Paulo, Brasil.

(4) Biólogo; Professor titular do Departamento de Fonoaudiologia da Faculdade de Filosofia e Ciências da Universidade Estadual Paulista "Julio de Mesquita Filho" (UNESP), Marília, São Paulo; Orientador do programa de Pós Graduação em Ciências da Reabilitação do Hospital de Reabilitação de Anomalias Craniofaciais da Universidade de São Paulo (HRAC-USP); Doutor em Anatomia Humana pela Faculdade de Ciências Médicas e Biológicas de Botucatu, São Paulo, Brasil.

(5) Biomédica; Professora titular do Departamento de Ciências Biológicas da Faculdade de Odontologia de Bauru da Universidade de São Paulo (FOB-USP) e do Laboratório de Fisiologia do Hospital de Reabilitação de Anomalias Craniofaciais da Universidade de São Paulo (HRAC-USP); Doutora em Fisiologia pela Faculdade de Medicina de Ribeirão Preto/USP, São Paulo, Brasil.

Conflito de interesses: inexistente 


\section{INTRODUÇÃO}

Indivíduos com fissura labiopalatina apresentam riscos de desenvolver alterações da comunicação oral as quais variam quanto à gravidade podendo levar a um prejuízo significativo da inteligibilidade da fala. Entre os distúrbios de fala que comumente são observados nas fissuras que envolvem o palato incluem-se: hipernasalidade, emissão de ar nasal, fraca pressão intra-oral e articulações compensatórias ${ }^{1-9}$.

A palatoplastia primária tem a finalidade de reparar anatômica e funcionalmente o palato, de modo a possibilitar o funcionamento adequado do esfíncter velofaríngeo e assim prevenir os sintomas de fala ${ }^{10,11}$. Para tanto, é preciso restabeler a função dos músculos palatinos assegurando que o palato fique com um comprimento adequado em relação à parede posterior da faringe. Além disso, as fibras musculares, tanto do músculo levantador quanto do músculo palatofaríngeo devem ser completamente liberadas de sua inserção no palato duro e reorientadas numa posição horizontal para criar a cinta muscular que possibilite a elevação do véu ${ }^{11,12}$.

Entretanto, apesar das tentativas de normalização da orientação muscular na correção primária do palato, uma parcela considerável de pacientes submetidos à palatoplastia primária permanece com a inserção muscular anteriorizada na borda posterior das lâminas palatinas, o que leva ao insucesso da palatoplastia primária persistindo a insuficiência velofaríngea (IVF) ${ }^{13}$. A proporção de IVF residual relatada na literatura varia entre os estudos ${ }^{14-16}$, sendo, então, necessário procedimento cirúrgico secundário.

Inúmeras técnicas cirúrgicas são descritas para a correção dos sintomas da IVF, tais como aumento da parede posterior da faringe, retroposição do palato (push-back), retalhos faríngeos, esfincteroplastias e mobilização dos músculos levantadores do palato. Neste último grupo inclui-se a veloplastia intravelar $7,10,12,17$ um procedimento que tem como objetivo reposicionar a musculatura do palato mole oferecendo boa mobilidade ao véu palatino e conseqüente melhora da competência velofaríngea. Sua utilização na palatoplastia secundária para correção da IVF residual está na dependência da anatomia e fisiologia da velofaringe identificadas na avaliação instrumental pré-cirúrgica e da experiência prévia do cirurgião ${ }^{12}$. As principais vantagens deste procedimento, do ponto de vista fisiológico, quando comparado a outras técnicas cirúrgicas, como o retalho faríngeo ou a esfincteroplastia estão no fato de proporcionar mobilidade ao palato mole, preservar a anatomia velofaríngea e, assim, não apresentar riscos de comprometimento das vias aéreas superiores.

Vários estudos demonstraram a eficiência da veloplastia intravelar na correção dos sintomas da fala decorrentes da IVF e têm defendido a realização deste procedimento como uma primeira tentativa para a correção da IVF',10,18-22.

O presente estudo teve por objetivo verificar - efeito da palatoplastia secundária realizada com veloplastia intravelar sobre a nasalidade e nasalância dos pacientes com fissura de palato reparada e insuficiência velofaríngea (IVF) e comparar os resultados entre estes pacientes, de acordo com o grau de fechamento velofaríngeo aferido na nasofaringoscopia.

\section{MÉTODO}

O estudo foi desenvolvido no Laboratório de Fisiologia do Hospital de Reabilitação de Anomalias Craniofaciais da Universidade de São Paulo (HRAC-USP). Os pacientes incluídos no estudo foram selecionados dentre aqueles com indicação para a palatoplastia secundária com veloplastia intravelar para correção da IVF. Não foram incluídos no estudo pacientes com síndromes e/ou problemas neurológicos evidentes e com sintomas respiratórios alérgicos agudos que resultassem em congestão nasal ao exame. Após serem informados sobre os objetivos do trabalho e os procedimentos aos quais seriam submetidos, em caso de aceite, o próprio paciente ou o seu responsável assinaram o termo de consentimento livre e esclarecido.

Foram avaliados 40 pacientes, de ambos os sexos, com idade entre 4 e 48 anos, com fissura de palato associada ou não à fissura de lábio, já submetidos às cirurgias primárias, que apresentavam IVF residual. Os pacientes foram divididos em dois grupos de acordo com a extensão da falha no fechamento velofaríngeo (gap velofaríngeo) aferida pela nasofaringoscopia pré-cirúrgica: grupo I, composto por 25 pacientes que apresentavam falhas pequenas e, grupo II, composto por 15 pacientes que apresentavam falhas grandes no fechamento velofaríngeo. A classificação do tamanho da falha velofaríngea foi obtida a partir do consenso da avaliação de três profissionais, sendo um cirurgião plástico e duas fonoaudiólogas que compõem a equipe de nasofaringoscopia do Hospital, todos eles com vasta experiência na realização destes exames os quais, aferiam a extensão do gap velofaríngeo, em conjunto, durante a realização do exame.

Todos os pacientes foram submetidos à avaliação perceptiva da fala e 39 pacientes foram submetidos à avaliação nasométrica, 3 dias antes 
e 8 meses, em média, após a palatoplastia secundária com veloplastia intravelar.

\section{Avaliação perceptiva da fala}

A avaliação perceptiva da fala foi realizada a fim de classificar a hipernasalidade aferida durante a conversa espontânea e repetição de lista de vocábulos e frases ${ }^{23}$. A hipernasalidade foi classificada em escala de 6 pontos, utilizando protocolo baseado em escores, onde 1=ausência de hipernasalidade (ressonância oronasal equilibrada), 2=hipernasalidade leve, 3=hipernasalidade leve para moderada, 4=hipernasalidade moderada, $5=$ hipernasalidade moderada para grave e $6=$ hipernasalidade grave. As avaliações foram realizadas por duas fonoaudiólogas da equipe do Laboratório, ambas com experiência de 15 e 26 anos, respectivamente, na avaliação de pacientes com fissura labiopalatina e insuficiência velofaríngea. As avaliações perceptivo-auditivas da fala foram realizadas individualmente, de modo presencial, em todos os pacientes que compõem a amostra do estudo.

\section{Avaliação Nasométrica da Fala}

A medida da nasalância foi determinada utilizando um nasômetro modelo 6200-3 IBM (Kay Elemetrics Corp, software versão 30-02-3.22). O exame foi realizado durante a leitura de um conjunto de cinco frases do português brasileiro, contendo sons exclusivamente orais (texto oral) para identificar hipernasalidade ${ }^{24}$, sendo considerado como limite superior de normalidade o escore de $27 \%$, ou seja, valores superiores a $27 \%$ foram considerados como sugestivos de hipernasalidade. Os indivíduos incapazes de ler o texto foram instruídos a repetir cada frase após o modelo do examinador. Variações individuais entre os valores pré e pós-operatórios foram consideradas clinicamente significativas quando superiores a 8 pontos perceptuais.

O presente estudo foi aprovado pelo Comitê de Ética em Pesquisa em Seres Humanos da Instituição sob o protocolo no 397/2006.

\section{Análise dos dados}

A significância das diferenças entre os escores obtidos no pré e pós-operatórios, relativos à hipernasalidade e à nasalância foi analisada pelo teste não paramétrico de Wilcoxon ${ }^{(25)}$. Na comparação entre os grupos utilizou-se o teste Mann-Whitney U. $\mathrm{Na}$ comparação de resolução e melhora entre os grupos utilizou-se o teste exato de Fisher. Foram aceitos como significantes os valores de $p<0,05$.

O sucesso da cirurgia foi analisado em função das seguintes observações nas avaliações pós-operatórias: 1) redução da hipernasalidade, definida como diminuição em um ou mais pontos em relação ao pré-operatório, sendo que este índice incorpora os casos de eliminação da hipernasalidade; 2) eliminação da hipernasalidade, definida como ausência da hipernasalidade; 3) redução da nasalância, definida como diminuição da nasalância maior ou igual a 8 pontos percentuais atingindo ou não o valor normal; 4) normalização da nasalância, definida como redução da nasalância para o valor normal $(\leq 27 \%)$.

\section{RESULTADOS}

\section{Avaliação perceptiva da fala}

Após a cirurgia, verificou-se no grupo I (falhas pequenas), redução da hipernasalidade em $84 \%$ (21/25) dos pacientes. Em 16\% (4/25) não houve alteração do sintoma. No grupo II (falhas grandes), a proporção de redução da hipernasalidade foi de $73 \%$ (11/15) e a proporção de pacientes sem alterações foi de $27 \%$ (4/15). A análise do sucesso cirúrgico de acordo com a avaliação perceptiva, para os grupos I e II, está ilustrada na figura 1.

Em termos de resolução do sintoma de fala, ou seja, eliminação da hipernasalidade, as proporções obtidas após a cirurgia foram consideravelmente menores. No grupo I, a eliminação da hipernasalidade foi observada em 64\% (16) dos pacientes e, proporção ainda menor, de $13 \%$ (2) foi obtida no grupo II, utilizando os mesmos critérios. Para ambos os grupos, não houve aumento da hipernasalidade.

A análise estatística mostrou que, em proporções significativamente maiores de pacientes do grupo I ocorreu eliminação da hipernasalidade em comparação com o grupo II.

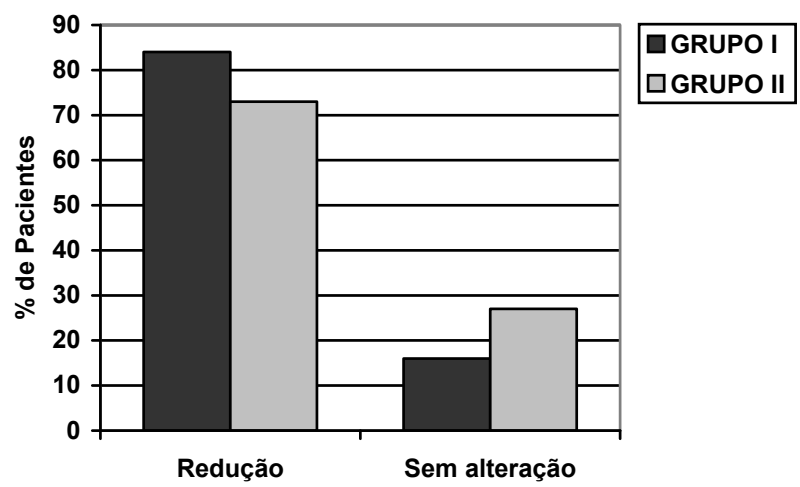

Figura 1 - Proporção de pacientes dos grupos I e II de acordo com o efeito da cirurgia sobre a hipernasalidade aferida perceptivamente: redução e sem alteração 


\section{Avaliação nasométrica}

O valor médio de nasalância obtido antes da cirurgia no grupo I foi de $36 \pm 11 \%$ e no grupo II de $41 \pm 7 \%$. Após a cirurgia, a nasalância média do grupo I diminuiu para $31 \pm 14 \%$ e a do grupo II permaneceu inalterada $41 \pm 15 \%$. As diferenças observadas não foram estatisticamente significantes em ambos os grupos. No entanto, a análise individual dos dados mostrou que, no pós-operatório, a proporção de pacientes em que se observou redução da nasalância no grupo | foi de $52 \%$ (13/25). Em 44\% (11/25) não se observou alteração da nasalância que fosse significativa clinicamente e, em 4\% (1/25) ocorreu aumento da nasalância, sugestivo de piora. Em 43\% (6/14) dos pacientes do grupo II ocorreu redução da nasalância e em $28 \%$ (4/14), a nasalância permaneceu inalterada. Aumento da nasalância, sugestivo de piora, ocorreu, igualmente, em $28 \%$ (4/14). A análise do sucesso cirúrgico de acordo com a avaliação nasométrica, para os grupos I e II, está ilustrada na figura 2.

Em termos de normalização verificou-se que $44 \%$ (11/25) dos pacientes do grupo I e 14\% (2/14) do grupo II passaram a apresentar valores de nasalância normais $(\leq 27 \%)$. Não houve diferença estatisticamente significante entre as proporções de redução e normalização da nasalância entre os dois grupos.

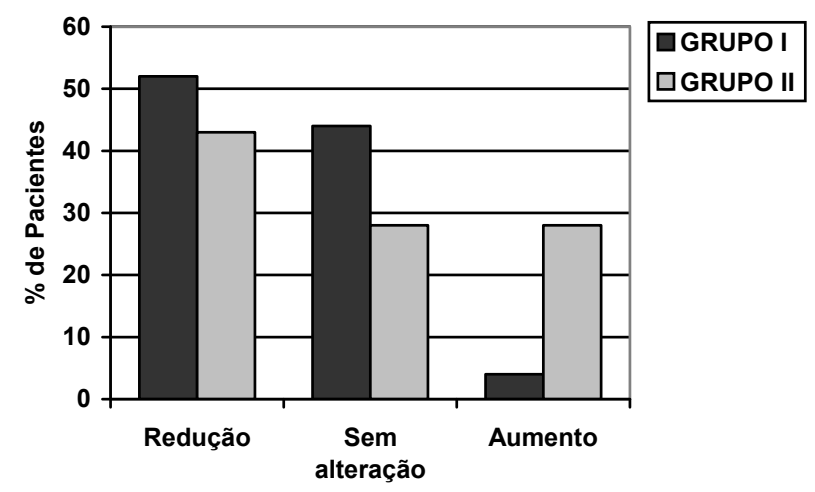

Figura 2 - Proporção de pacientes dos grupos I e II de acordo com o efeito da cirurgia sobre a nasalância: redução, sem alteração ou aumento dos valores

\section{DISCUSSÃO}

Cada vez mais a veloplastia intravelar vem sendo empregada com sucesso na correção da IVF residual pelo fato de proporcionar uma condição favorável à mobilidade velofaríngea e por ser um procedimento com menor risco de morbidade quando comparado aos demais procedimentos secundários como o retalho faríngeo e a esfincteroplastia. Os principais critérios a serem considerados na indicação da veloplastia intravelar relacionam-se à inserção anterior da musculatura do véu palatino ${ }^{2}$ e à pequena extensão da falha no fechamento velofaríngeo (gap velofaríngeo), que, segundo alguns autores não deve ultrapassar $5 \mathrm{~mm}^{18}$. Embora a inserção anterior da musculatura velar não seja a única causa da IVF, uma vez identificada, tornase fundamental reposicionar o músculo levantador do véu palatino para uma orientação mais posterior, recriando, assim, a cinta muscular que, teoricamente, favorece os movimentos de elevação e deslocamento posterior do véu $u^{2,12}$.

É fato que a veloplastia intravelar é indicada nos casos com gap velofaríngeo pequeno. Entretanto, estudos têm demonstrado que tal procedimento é, também, efetivo na presença de IVF grave. Em um estudo $^{2}$ que incluiu pacientes com gaps pequenos, médios e grandes, tal como no presente estudo, os autores verificaram que 6 pacientes com gap velofaríngeo médio e $1 \mathrm{com}$ gap grande obtiveram completa resolução da IVF após a veloplastia intravelar secundária. No presente estudo, eliminação da hipernasalidade foi obtida somente em 13\% (2) dos pacientes grupoll.

Por outro lado, outros autores ${ }^{18}$ verificaram que, pacientes que apresentavam gaps maiores permaneceram com IVF após a palatoplastia secundária. Embora os sintomas de fala tenham melhorado, o fechamento velofaríngeo completo não foi alcançado. Uma vez que o objetivo da cirurgia secundária para correção da IVF é restaurar a anatomia normal ou modificar a anatomia existente para melhorar a função velofaríngea, é esperado algum benefício, (ainda que não solucione completamente os sintomas), também nos pacientes que apresentam falhas médias e grandes do fechamento velofaríngeo. Estes pacientes, por terem adquirido uma melhor condição velofaríngea, poderão se beneficiar, no futuro, de outras intervenções cirúrgicas mais favoráveis ${ }^{2}$.

No presente estudo, pretendeu-se verificar o efeito deste procedimento sobre a nasalidade de pacientes com fissura de palato reparada e IVF residual decorrente de falhas grandes e pequenas no fechamento velofaríngeo, por meio de metodologia perceptiva combinada à nasométrica, utilizando critérios bem definidos de sucesso cirúrgico. Os resultados foram analisados em termos de redução dos sintomas de hipernasalidade e nasalância e em termos de resolução completa ou normalização dessas variáveis.

De modo geral, foi possível verificar que a cirurgia levou à melhora dos sintomas de fala na maioria dos pacientes nos dois grupos estudados. 
Para a hipernasalidade, por exemplo, a melhora ocorreu em $84 \%$ dos pacientes do grupo com falhas pequenas e em $73 \%$ dos pacientes com falhas maiores no fechamento velofaríngeo.

Proporções semelhantes às do grupo I foram relatadas por outros autores ${ }^{19,20}$ que verificaram melhora em $87 \%$ e $82 \%$ dos pacientes, respectivamente, ao estudarem o efeito da veloplastia intravelar sobre a hipernasalidade em pacientes com gap velofaríngeo pequeno. Isto reforça o que já está bem estabelecido na literatura no que se refere à indicação da veloplastia intravelar para pacientes com IVF mais leve. É evidente que a reduzida morbidade da veloplastia intravelar torna este procedimento uma opção de tratamento potencial para pacientes com menor grau de IVF que, embora sintomática, pode não ser grave o suficiente para justificar a indicação de um retalho faríngeo.

A menor proporção de pacientes com redução dos sintomas encontrada no grupo II se justifica, provavelmente, pela condição pré-operatória, já que apresentavam falhas grandes no fechamento velofaríngeo. A indicação da veloplastia intravelar nestes casos parte do pressuposto que tal procedimento reorganiza a musculatura do véu palatino sendo, então, considerada uma primeira tentativa de minimizar os sintomas da IVF. Mesmo não havendo a resolução completa dos sintomas de fala, esta condição velofaríngea favorável pode evitar a indicação de procedimentos futuros que comprometem as vias aéreas superiores, como por exemplo, o retalho faríngeo muito largo ${ }^{2,19}$. Isto foi comprovado no presente estudo ao verificarmos que em $73 \%$ dos pacientes do grupo II a cirurgia levou à redução da hipernasalidade.

A literatura tem defendido que, enquanto a veloplastia intravelar restaura a anatomia velofaríngea normal e ainda redireciona os músculos do palato na posição transversa, o retalho faríngeo rompe a integridade anatômica da musculatura velofaríngea criando uma ponte entre o palato e a parede posterior da faringe que pode levar à obstrução das vias aéreas superiores e à hiponasalidade e por isto é considerado "não-fisiológico". Em estudo conduzido no laboratório de Fisiologia ${ }^{(1)}$, verificou-se que $35 \%$ dos pacientes submetidos ao retalho faríngeo desenvolveram hiponasalidade iatrogênica. Embora o retalho seja um procedimento cirúrgico efetivo para a correção de IVF grave ${ }^{26}$, é inquestionável seu potencial significante de comprometimento das vias aéreas superiores. Em outro estudo desenvolvido neste mesmo laboratório ${ }^{27}$, foi demonstrado que $36 \%$ dos pacientes submetidos ao retalho faríngeo passaram a relatar queixas respiratórias tais como respiração oral, ronco e sensação de dificuldade respiratória durante o sono, após a cirurgia. A esfincteroplastia, embora menos danosa à anatomia normal da velofaringe, também pode levar à obstrução respiratória ${ }^{12,18}$.

A fim de complementar o resultado obtido na avaliação perceptiva da fala, no presente estudo foi utilizado também, a nasometria (método instrumental) para a análise dos resultados cirúrgicos. Muito embora, em média, não se tenha observado diferença entre os valores pré e pós-operatórios, a análise individual dos dados mostrou que a cirurgia levou à melhora e, até mesmo, à resolução da nasalância em parcela considerável de pacientes. Em $52 \%$ dos pacientes do grupo I e em $43 \%$ dos pacientes do grupo II a cirurgia levou à redução da nasalância e, em $44 \%$ no grupo I e $14 \%$ no grupo II houve normalização dos escores de nasalância. Outros autores ${ }^{20}$ relataram proporções bastante superiores, $87 \%$ de melhora e $56 \%$ de normalização da função velofaríngea avaliada, também, por meio da nasometria. Entretanto, seus resultados não são comparáveis aos do presente estudo, pois, direfentemente do presente estudo, que utilizou a leitura de um texto contendo 5 frases com sons exclusivamente orais, estes autores utilizaram a emissão de vogal alta isolada e de sílaba contendo vogal alta para determinar a nasalância. No que se refere à melhora da nasalância, os resultados obtidos no presente estudo são, também, ligeiramente inferiores aos resultados obtidos em pacientes submetidos ao retalho faríngeo ${ }^{1,28}$ os quais verificaram $60 \%$ e $68 \%$ de redução de nasalância no texto oral, respectivamente. Por outro lado, os resultados obtidos no grupo I foram semelhantes no que se refere à normalização da nasalância, 35\% e 55\%, respectivamente.

Ainda que o método instrumental tenha confirmado os achados da avaliação perceptiva, a nasometria mostrou-se sensível, também, para detectar piora (aumento da nasalância) em 1 paciente do grupo I e 1 paciente do grupo II, não constatadas na avaliação subjetiva. $O$ aumento da nasalância já foi verificado em 11 de 241 pacientes submetidos ao retalho faríngeo em outro estudo realizado no labortório de Fisiologia ${ }^{28}$. O aumento da nasalância não é um resultado esperado após a veloplastia intravelar. A princípio, este aumento pode ser explicado como conseqüência de uma "piora" da função velofaríngea decorrente, talvez, de um aumento do gap velofaríngeo devido à retração cicatricial após a cirurgia. Estudos posteriores devem ser conduzidos para avaliar a persistência destes resultados a longo prazo. 


\section{CONCLUSÃO}

Concluiu-se que a cirurgia levou à melhora da fala em parcela considerável de pacientes, num prazo inferior a um ano, especialmente naqueles que apresentavam falhas pequenas do fechamento velofaríngeo. A cirurgia foi eficiente também, embora em menor proporção, nos pacientes com falhas grandes do fechamento velofaríngeo. Estes, por sua vez, são os casos que poderão ser beneficiados, no futuro, com outro procedimento cirúrgico por terem adquirido uma condição estrutural mais favorável ao fechamento velofaríngeo.

\begin{abstract}
Purpose: to check the effect of secondary palatoplasty performed with intravelar veloplasty on the nasality and nasalance of patients with repaired cleft palate and velopharyngeal insufficiency (VPI) and compare the outcome among these patients, according to the degree of velopharyngeal closure as analyzed by nasopharyngoscopy. Method: prospective study with 40 patients of both genders, aged from 4 to 48 years, with repaired cleft palate and residual VPI, evaluated for 3 days before and 8 months after palatoplasty, on average, divided into two groups: one with 25 patients with minor defect (group I) and the other with 15 patients with medium to large defect (group II) in velopharyngeal closure. Hypernasality was evaluated perceptually and nasalance was assessed by nasometry. Differences between groups and stages were considered to be significant at the $5 \%$ level. The local Ethics Committee for Human Research approved the study. Results: after surgery, we observed a reduction of hypernasality in $84 \%$ of the patients from group I, and in $73 \%$ of the patients from group II. Reduction of nasalance scores were observed in $52 \%$ of the group I cases and in $43 \%$ of the group II. Conclusion: secondary palatoplasty performed with intravelar veloplasty led to improvement in nasality in most of the analyzed patients. The results further demonstrated that surgery was more effective in patients with minor defects in velopharyngeal closure.
\end{abstract}

KEYWORDS: Speech; Cleft Palate; Velopharyngeal Insufficiency

\section{REFERÊNCIAS}

1. Zuiani TBB, Trindade IEK, Yamashita RP, Trindade Junior AS. The pharyngeal flap surgery in patients with velopharyngeal insufficiency: perceptual and nasometric speech assessment. Braz J Dysmorphol Speech Disord. 1998; 2(1):31-42.

2. Sie KCY, Tampakopoulou DA, Sorom JBA, Gruss JS Eblen LE. Results with Furlow palatoplasty in management of velopharyngeal insufficiency. Plast Reconstr Surg. 2001; 108(1):17-25.

3. Sie 2006. Cleft palate speech and velopharyngeal insufficiency: surgical approach. B-ENT. 2006; 2 Suppl 4:85-94.

4. Yamashita RP, Calais LL, Miguel HC, Trindade IEK. Avaliação da resistência laríngea em indivíduos portadores de insuficiência velofaríngea com distúrbio articulatório compensatório. Acta ORL. 2006; 24(4):263-7.

5. Genaro KF, Fukushiro AP, Suguimoto MLFCP. Avaliação e Tratamento dos distúrbios da fala. In: Trindade IEK, Silva Filho OG, organizadores.
Fissuras labiopalatinas: uma abordagem interdisciplinar. São Paulo: Santos; 2007. p.109-22. 6. Smith BE, Kuehn DP. Speech Evaluation of Velopharyngeal Dysfunction. J Craniofac Surg. 2007; 18(2):251-61.

7. Sie KC, Chen EY. Management of Velopharyngeal Insufficiency: Development of a Protocol and Modifications of Sphincter Pharyngoplasty. Facial Plast Surg. 2007; 23(2):128-39.

8. Henningsson G, Kuehn DP, Sell D, Sweeney T, Trost-Cardamone JE, Whitehill TL; Speech Parameters Group. Universal parameters for reporting speech outcomes in individuals with cleft palate. Cleft Palate Craniofac J. 2008; 45(1):2-17.

9. RudnickEF, Sie KC. Velopharyngeal insufficiency: current concepts in diagnosis and management. Curr Opin Otolaryngol Head Neck Surg. 2008; 16(6):530-5.

10. Noorchashm N, Dudas J.R, Ford M, Gastman B, Deleyiannis F W-B, Vecchione L, Jiang $S$, Cooper G. M, Haralam M A, Losee J E. Conversion Furlow Palatoplasty Salvage of Speech After Straight-Line 
Palatoplasty and "Incomplete Intravelar Veloplasty". Annals of Plastic Surgery. 2006; 56(5):505-10.

11. Andrades $\mathrm{P}$, Monteros AE, Shell $\mathrm{DH}$, Thurston TE, Fowler HS, Xavier ST et al. The importance of radical intravelar veloplasty during two-flap palatoplasty. Plast Reconstr Surg. 2008; 122(4):1121-30.

12. Huang MHR, Lee ST, Rajendran K. Anatomic basis of cleft palate and velopharyngeal surgery: implications from a fresh cadaveric study. Plast Reconstr Surg 1998; 101(3):613-27.

13. Mink van der Molen $A B$, Janssen K, Specken TF, Stubenitsky BM. The modified Honig velopharyngoplasty - a new technique to treat hypernasality by palatal lengthening. $J$ Plast Reconstr Aesthet Surg. 2009; 62(5):646-9.

14. Phua YS, de Chalain T. Incidence of oronasal fistulae and velopharyngeal insufficiency after cleft palate repair: an audit of 211 children born between 1990 and 2004. Cleft Palate Craniofac J. 2008; 45(2):172-8

15. Koh KS, Kang BS, Seo DW. Speech evaluation after repair of unilateral complete cleft palate using modified 2-flap palatoplasty. J Craniofac Surg. 2009; 20(1):111-4; discussion 115.

16. Nyberg J, Westberg LR, Neovius E, Larson O, Henningsson G. Speech Results After One-Stage Palatoplasty With or Without Muscle Reconstruction for Isolated Cleft Palate. Cleft Palate Craniofac J. 2010; 47(1):92-103.

17. Rocha DL. Tratamento cirúrgico da insuficiência velofaríngea. In: Trindade IEK, Silva Filho OG, organizadores. Fissuras labiopalatinas: uma abordagem interdisciplinar. São Paulo: Santos; 2007. p. 145-63.

18. Chen PTK, Wu JTH, Chen YR, Noordhoff S. Correction of secundary velopharyngeal insufficiency in cleft palate patients with the Furlow palatoplasty. Plast Reconstr Surg. 1994; 94(7):933-41.

19. Sommerlad BC, Wehendale FV, Birch MJ, Sell D, Hattee C, Harland K. Palate re-repair revisited. Cleft Palate Craniofac J. 2002; 39(3):295-307.
20. Nakamura N, Ogata Y, Sassaguri M, Suzuki A, Kilkuta R, Ohishi M. Aerodynamic and cephalometric analysis of velopharyngeal structure and function following repushback surgery for secondary correction in cleft palate. Cleft Palate Craniofac J. 2003; 40(1):46-53.

21. Hassan ME, Askar S. Does palatal muscle reconstruction affect the functional outcome of cleft palate surgery? Plast Reconstr Surg. 2007; 119(6):1859-65.

22. Smolka K, Seifert E, Eggensperger N, lizuka T, Smolka W. Reconstruction of the palatal aponeurosis with autogenous fascia lata in secondary radical intravelar veloplasty: a new method. Int $\mathrm{J}$ Oral Maxillofac Surg. 2008; 37(8):1-5.

23. Genaro KF, Yamashita RP, Trindade IEK. Avaliação clínica e instrumental na fissura labiopalatina. In: Fernandes FDM, Mendes BCA, Navas ALPGP (org.). Tratado de Fonoaudiologia. São Paulo, Roca. 2009. p. 488-503.

24. Trindade IEK, Genaro KF, Dalston RM. Nasalance scores of normal brasilian portuguese speakers. Braz J Dysmorphol Speech Hear Disord. 1997; 1(1):23-34.

25. Zar JH. Biostatistical analysis. 3rd ed. New Jersey: Prentice-Hall; 1996.

26. Dailey SA, Karnell MP, Karnell LH, Canady JW. Comparison of resonance outcomes after pharyngeal flap and Furlow double-opposing z-plasty for surgical management of velopharyngeal incompetence. Cleft Palate Craniofac J. 2006; 43(1):38-43.

27. Yamashita RP, Trindade IEK. Long-term effects of pharyngeal flaps on the upper airways of subjects with velopharyngeal insufficiency. Cleft Palate Craniofac J. 2008; 45(4):364-70.

28. Fukushiro AP. Análise perceptiva, nasométrica e aerodinâmica da fala de indivíduos submetidos à cirurgia de retalho faríngeo para a correção da insuficiência velofaríngea [tese]. Bauru: Hospital de Reabilitação de Anomalias Craniofaciais, Universidade de São Paulo; 2007.
http://dx.doi.org/10.1590/S1516-18462011005000040

RECEBIDO EM: 22/09/2010

ACEITO EM: 27/01/2011

Endereço para correspondência:

Dra. Renata Paciello Yamashita

Laboratório de Fisiologia do HRAC-USP

Rua Silvio Marchione, 3-20

Bauru - SP

CEP: $17012-900$

E-mail: rezeyama@usp.br 